
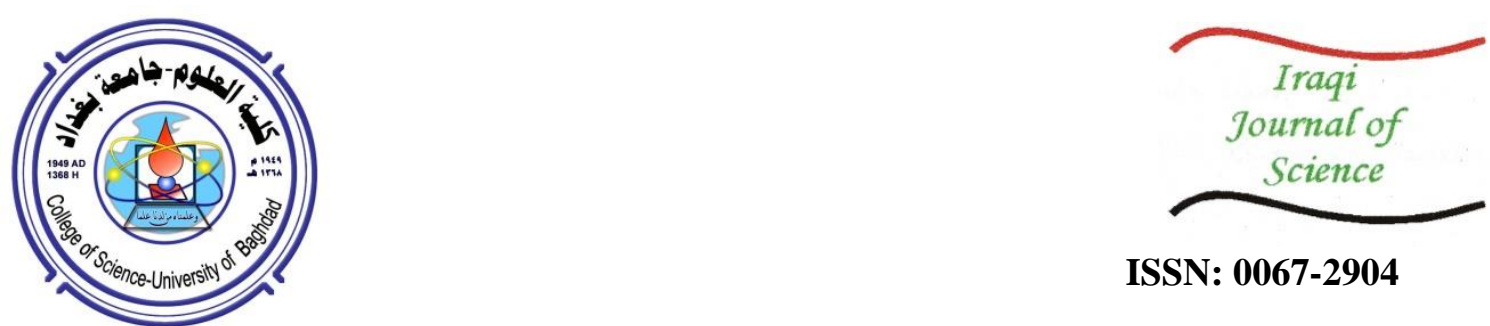

ISSN: 0067-2904

\title{
Some Geometric Properties of a Hyperbolic Univalent Function
}

\author{
Sabah S. Al-Azawee, Shatha S. Alhily *
}

Department of Mathematics, College of Sciences, Mustansiriyah University, Baghdad, Iraq

$$
\text { Received: } 25 / 12 / 2020
$$

Accepted: 20/3/2021

\begin{abstract}
In this paper, we analyze several aspects of a hyperbolic univalent function related to convexity properties, by assuming $f$ to be the univalent holomorphic function maps of the unit disk $\mathfrak{D}=\{z \in \mathbb{C}:|z|<1\}$ onto the hyperbolic convex region $\mathfrak{L}(\mathfrak{L}$ is an open connected subset of $\mathbb{C})$. This assumption leads to the coverage of some of the findings that are started by seeking a convex univalent function distortion property to provide an approximation of the inequality $\frac{f^{\prime \prime}(z)}{f^{\prime}(z)}-$ $\left.\frac{\mathfrak{r}}{1-\mathfrak{r}^{2}}\left|\leq \frac{2}{1-\mathfrak{r}^{2}}\right| f^{\prime}(z)\right|^{-1}$ and confirm the form of the lower bound for $|f(z)| \leq \frac{\mathrm{r}}{1-\mathrm{r}}$. A further result was reached by combining the distortion and growth properties for increasing inequality $\left|\frac{z f^{\prime}(z)}{f(z)}\right| \geq \frac{1}{1+r}$. From the last result, we wanted to demonstrate the effect of the unit disk image on the condition of convexity estimation by proving the two inequalities of

$\left|\frac{f^{\prime \prime}(z)}{z f^{\prime}(z)}\right|<\frac{2 \mathfrak{r}}{1-\mathfrak{r}^{2}} \quad$ on $f(\mathfrak{D})=\mathfrak{L}$, and $\quad\left|\frac{f^{\prime \prime}(z)}{z f^{\prime}(z)}\right|<1 \quad$ on $\mathfrak{L}=\mathfrak{D}$.
\end{abstract}

Keywords: univalent function, convex function, convex region, hyperbolic metric space.

\section{Introduction}

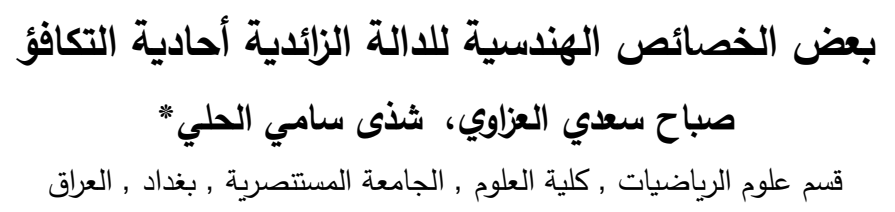

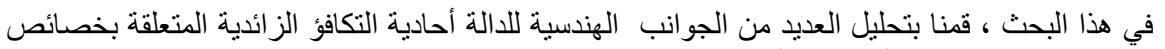

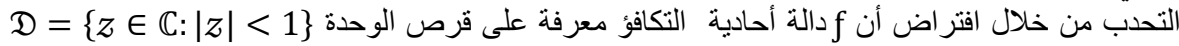

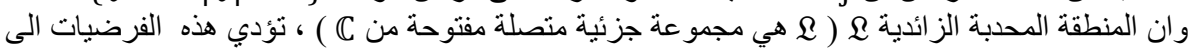

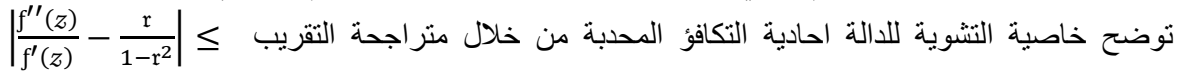

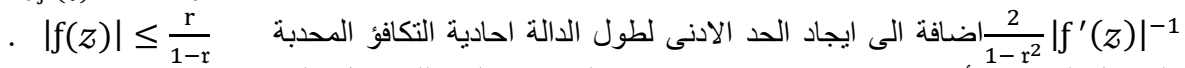

و التوصل إلى نتيجة أخرى تجمع بين خواص نظريتي التشويه و النمو للحصول على متر اجحة متز ايده التها. من $\left|\frac{z f^{\prime}(z)}{f(z)}\right| \geq \frac{1}{1+r}$

التحدب من خلال إثبات وجود مثر اجنتين اساسية لبيان ناثثير الصفة الهندسية للتحدب في الدالة احادية التكافؤ $\mathfrak{L}=\mathfrak{D}$ عندما $\left|\frac{f^{\prime \prime}(z)}{z f^{\prime}(z)}\right|<1$ وكذللك $\left|\frac{f^{\prime \prime}(z)}{z f^{\prime}(z)}\right|<\frac{2 \mathfrak{r}}{1-\mathfrak{r}^{2}}$

The typical problem in the Geometric Function Theory has always been to maximize the value of a particular function over a given class of analytical functions. This class contains a single valued function in a domain $\mathfrak{L} \subset \mathbb{C}$ that is called univalent function, if it ever never takes the same values 
twice (one to one). We shall be concerned with the class $\mathcal{S}$ of a holomorphic and univalent function in the unit disk $\mathfrak{D}=\{z \in \mathbb{C}:|z|<1\}$, normalized under two conditions, $f(0)=0, f^{\prime}(0)=1$, where each $f \in \mathcal{S}$ can be represented by a Taylor series of the form $f(z)=\sum_{m=0}^{\infty} a_{m} z^{m}$. The subclass of $\mathcal{S}$ consists of the convex function (which is one of those functions that maps the disk to a convex domain conformally, which is denoted by $\mathcal{C}[1,2]$

The class $\mathcal{P}$ of all functions $f$ that are holomorphic and have positive real part is closely related to both classes $\mathcal{S}$ and . The most famous case of this relation is the Bieberbach conjecture of achieving the full co-efficiency of the expansion of the univalent function of the power series for another important problem, namely the distortion (cf. [3]).

Here, we are dealing with hyperbolic univalent functions with property of convexity. These functions attracted a great deal of interest, especially in recent years, as they were applied to surfaces and certain types of classes (cf. [4-6].

In 1987, Minda [7] published one of the first papers on hyperbolic convexity of univalent functions, and in 1994, Ma and Minda [8] provided the first general description of hyperbolically convex functions on growth problems. In that same year [9], Kim and Minda reached two-point theorems for convex regions. These theorems are a comparative analysis between hyperbolic geometry and Euclidean geometry. In [10], the authors also speculated that the Schwarzian derivative is maximized by the hyperbolic strip map, whereas Roger et al. recently showed this relation [11]. The study of twopoint distortion theorems for an univalent function on a unit disk, through the definition of hyperbolic metric by theorem, provides a necessary condition for simply connected regions on a complex plane [12]. Yamashita [9] also used a metric to define several aspects for convex regions.

In 2000, Mejia and Pommerenke [13] started working on hyperbolically convex functions. They stated that the Schwarzian derivative was maximized by the hyperbolic strip mapping.

In 2017, Alhily [14] showed some results on the function representation of the convexity area for univalent function by applying the weighted composition operator to the convexity of the Bergman spaces.

It is now important to remember that the hyperbolic plane is formed from the unit disk $\mathfrak{D}=$ $\{z \in \mathbb{C}:|z|<1\}$ and the hyperbolic metric. The hyperbolic metric on the unit disk $\mathfrak{D}$ is defined by $\kappa_{\mathfrak{D}}(z)|\mathrm{d} z|=\frac{|\mathrm{d} z|}{1-|z|^{2}}$. Also, we need to know certain properties about the hyperbolic geodesic arc $\gamma$ in $\mathfrak{D}$, which joins the two points $z_{1}$ and $z_{2}$ and is orthogonal to the unit circle in a certain sub-region $\mathfrak{L} \subset \mathfrak{D}$, that is called hyperbolic distance.

$d_{\mathfrak{D}}\left(z_{1}, z_{2}\right)=\inf _{\gamma} \mathcal{L}_{\mathfrak{D}}(\gamma)$, where $\mathcal{L}_{\mathfrak{D}}(\gamma)=\int_{\gamma} \kappa_{\mathfrak{D}}(z)|\mathrm{d} z|$.

One can perceive another geometric concept, which is the hyperbolic metric density on the hyperbolic region, that played the major role in the development of the classical geometric theory, which is defined in the form that a convex set or a convex region is a subset that intersects every line into a single line segment,

$\kappa_{\mathfrak{L}}[f(z)]\left|f^{\prime}(z)\right|=\kappa_{\mathfrak{D}}(z)$,

where $f$ is a holomorphic generic covering projection of $\mathfrak{D}$ onto $\mathfrak{L}$.

\section{Preliminaries}

\section{Definition (1.1) [Gaussian curvature] $[15,16]$}

Curvature is an integrated part of the curve that defines its geometry at a point.

i. The formula $h=\left|\frac{d \mathcal{T}}{d s}\right|$ shows how quickly the unit tangent vector rotates at a certain point, where $\mathcal{T}$ is the vector of the unit tangent and $d s$ is the differential of the length of the curve.

ii. The formula $h=\frac{|v \times a|}{|v|^{3}}$ represents the curve in the direction of a moving point and is determined by time $t$, where $v$ is the velocity, $a$ is the acceleration, and " $\times$ " is the symbol of the vector product.

iii. The formula $h_{\mathfrak{Q}}(z, \gamma)=h_{\mathrm{e}}(z, \gamma)-\frac{\partial \log h_{\mathfrak{R}}(z)}{\partial n(z)}$

$$
=h_{\mathrm{e}}(z, \gamma)+2 \mathfrak{J}\left[\frac{\partial \log h_{\Omega}(z)}{\partial z} \cdot \frac{z^{\prime}(t)}{\left|z^{\prime}(t)\right|}\right],
$$

be Gaussian curvature where $h_{\mathrm{e}}(z, \gamma)$ is the euclidean curvature with the unit normal $n(z)$ at $z$ that makes an angle with tangent vector, which is $\frac{\pi}{2}$. 


\section{Definition (1.2) [ Convex univalent function] [ 3]}

Let $f$ be a holomorphic and univalent function in the unit disk $\mathfrak{D}=\{z \in \mathbb{C}:|z|<1\}$ and maps the unit disk onto a convex domain. Then, $f$ is said to be convex univalent function (or simple convex).

\section{Definition (1.3) [ Hyperbolic convex function] [8]}

A holomorphic and univalent function $f$ in the unit disk $\mathfrak{D}=\{z \in \mathbb{C}:|z|<1\}$ is called hyperbolic convex if the image region is a hyperbolically convex subset of $\mathfrak{D}$.

Theorem (1.1) [ 1 ]. Suppose that $\mathfrak{L}$ is a convex hyperbolic region in $\mathbb{C}$. Then, for all $\mathcal{A}, \mathcal{B} \in \mathfrak{L}$. $\mathrm{e}^{-2 \mathrm{~d}_{\mathfrak{L}}(\mathcal{A}, \mathcal{B})} \leq \frac{\kappa_{\mathcal{L}}(\mathcal{B})}{\kappa_{\mathfrak{R}}(\mathcal{A})} \leq \mathrm{e}^{2 \mathrm{~d}_{\mathfrak{L}}(\mathcal{A}, \mathcal{B})}$, equality holds if and only if $\mathfrak{L}$ is a half plane and the line segment joining $\mathcal{A}$ and $\mathcal{B}$ is perpendicular to the boundary of $\mathfrak{L}$.

\section{Results}

Here are some interesting results for the classical distortion and growth properties for the convex univalent function.

Theorem (2.1). Let $f: \mathfrak{D} \rightarrow \mathfrak{L}$ be a convex univalent function, where $\mathfrak{L}$ is a hyperbolic region. Then, $\left|\frac{f^{\prime \prime}(z)}{f^{\prime}(z)}-\frac{r}{1-\mathrm{r}^{2}}\right| \leq \frac{2}{1-\mathrm{r}^{2}}\left|f^{\prime}(z)\right|^{-1}$.

Proof. Given that $f$ is a univalent and convex function which belongs to $\mathcal{S}$, that is $f(0)=0$ and $f^{\prime}(0)=1$.

Let $\mathfrak{L}$ be a convex domain and $z_{1}, z_{2}$ are in $\mathfrak{L}$ and joined by the curve $\gamma$.

Now, substitute $f(\zeta), f(z)$ for $z_{1}, z_{2}$ respectively, such that $f(\mathfrak{D})=\mathfrak{L}$.

Then, $\kappa_{\mathfrak{L}} f(z)\left|f^{\prime}(z)\right|=\kappa_{\mathfrak{D}}(z)$,

where $\kappa_{\mathfrak{D}}(z)=\frac{1}{1-|z|^{2}}$. Hence this will imply that $K_{\mathfrak{L}}(f(z))\left|f^{\prime}(z)\right|=\frac{1}{1-|z|^{2}}$.

Apply the logarithm function to the earlier statement, as follow

$$
\begin{gathered}
\log \left[\kappa_{\mathfrak{L}}(f(z)) \cdot\left|f^{\prime}(z)\right|\right]=\log \frac{1}{1-|z|^{2}} \\
\log \kappa_{\mathfrak{L}}(f(z))+\log \left|f^{\prime}(z)\right|=\log \frac{1}{1-|z|^{2}}
\end{gathered}
$$

Derive both sides to the earlier statement to obtain

$$
\begin{aligned}
& \frac{\partial}{\partial w}\left(\log \Lambda_{\mathfrak{L}}(f(z))\right)+\frac{\partial}{\partial w}\left(\log \left|f^{\prime}(z)\right|\right)=\frac{\partial}{\partial z}\left(\log \frac{1}{1-|z|^{2}}\right) \\
& \frac{\partial}{\partial w}\left(\log \Lambda_{\mathfrak{L}}(f(z))\right)+\left|\frac{f^{\prime \prime}(z)}{f^{\prime}(z)}\right|=\frac{\bar{z}}{1-|z|^{2}} \\
& \left|\frac{\partial}{\partial w}\left(\log \Lambda_{\mathfrak{L}}(f(z))\right)+\frac{f^{\prime \prime}(z)}{f^{\prime}(z)}\right|=\frac{\bar{z}}{1-|z|^{2}} \\
& \left|\frac{\partial}{\partial w}\left(\log \Lambda_{\mathfrak{L}}(f(z))\right)+\frac{f^{\prime \prime}(z)}{f^{\prime}(z)}\right|=\frac{\mathfrak{r}}{1-\mathfrak{r}^{2}} \\
& \left|\frac{\partial}{\partial w}\left(\log \Lambda_{\mathfrak{L}}(f(z))\right)+\frac{f^{\prime \prime}(z)}{f^{\prime}(z)}\right| \leq\left|\frac{\partial}{\partial w} \log \left(K_{\mathfrak{L}}(f(z))\right)\right|+\left|\frac{f^{\prime \prime}(z)}{f^{\prime}(z)}\right| \\
& \leq 2 \kappa_{\mathfrak{L}}(f(z))+\left|\frac{f^{\prime \prime}(z)}{f^{\prime}(z)}\right| \text {. }
\end{aligned}
$$

Since $\frac{\partial}{\partial w} \log \Lambda_{\mathfrak{L}}[f(z)] \leq 2 \Lambda_{\mathfrak{L}}(f(z))$ is a Gaussian Curvature, then we can apply inequality (2.2) in order to obtain

$$
\frac{\mathfrak{r}}{1-\mathfrak{r}^{2}} \leq 2 \kappa_{\mathfrak{L}}(f(z))+\left|\frac{f^{\prime \prime}(z)}{f^{\prime}(z)}\right|
$$

In the last step, we make a short calculation of inequality (3.2) with the use of inequality (1.2) to obtain

The proof is complete

$$
\left|\frac{f^{\prime \prime}(z)}{f^{\prime}(z)}-\frac{\mathfrak{r}}{1-\mathfrak{r}^{2}}\right| \leq \frac{2}{1-\mathfrak{r}^{2}}\left|f^{\prime}\right|^{-1}
$$

Theorem (2.2) If $f$ is a univalent and convex function on a convexity region $\mathfrak{L}$ such that $f(0)=0$, then $|f(z)| \leq \frac{\mathfrak{r}}{1-\mathfrak{r}}, \mathfrak{r}=|z|$. 
Proof . Let $\exp \left({ }^{-2 \mathrm{~d}_{\mathfrak{L}}(\mathcal{A}, \mathrm{B})}\right) \leq \frac{\kappa_{\mathfrak{L}}(\mathcal{B})}{\kappa_{\mathfrak{R}}(\mathcal{A})} \leq \exp \left({ }^{2 \mathrm{~d}_{\mathfrak{L}}(\mathcal{A}, \mathrm{B})}\right)$,

be a statement that is provided by theorem $(1.1)$, where $\mathcal{A}, \mathcal{B}$ are two points that belong to $\mathfrak{R}$.

Let us start with the left- hand side of (2.4), as follow

$\frac{\kappa_{\mathfrak{R}}(\mathcal{B})}{\kappa_{\mathfrak{R}}(\mathcal{A})} \geq \exp \left[-2 \mathrm{~d}_{\mathfrak{R}}(\mathcal{A}, \mathcal{B})\right]$, such that

$\kappa_{\mathfrak{L}}(\mathcal{B}) \geq \kappa_{\mathfrak{L}}(\mathcal{A}) \exp \left[-2 \mathrm{~d}_{\mathfrak{L}}(\mathcal{A}, \mathcal{B})\right]$

$$
\frac{1}{\Lambda_{\mathfrak{L}}(\mathcal{B})} \leq \frac{1}{\Lambda_{\mathfrak{L}}(\mathcal{A}) \exp \left[-2 \mathrm{~d}_{\mathfrak{L}}(\mathcal{A}, \mathcal{B})\right]}
$$

It is regarded that $\Lambda_{\mathfrak{L}}(\mathcal{B}) \equiv \Lambda_{\mathfrak{L}}(\mathrm{w}(\mathrm{s}))$, since $\mathrm{w}(\mathrm{s}) \subset \gamma: \mathcal{A} \rightarrow \mathcal{B}$ for all $\mathrm{s} \in[0, \ell]$.

Therefore, it must be assumed that $f(z)$ for $\mathcal{A}$ and 0 for $\mathcal{B}$, where $f(s=0)=0$, since ( $f$ is univalent function on $\mathfrak{L})$, in order to get

$$
\begin{aligned}
|f(z)| & =\int_{0}^{\ell} \frac{d s}{\kappa_{\mathfrak{L}}(f(s))} \leq \int_{0}^{\ell} \frac{\exp \left[2 d_{\mathfrak{L}}(f(s), f(s=0))\right]}{\kappa_{\mathfrak{L}}(f(s))} \\
& =\frac{1}{\kappa_{\mathfrak{L}}(\mathcal{A})}\left[\frac{1}{2} \int_{0}^{\ell} \exp \left[2 d_{\mathfrak{L}}(f(s), f(s=0))\right] d s\right] \\
& =\frac{1}{\Lambda_{\mathfrak{L}}(\mathcal{A})}\left[\left.\frac{1}{2} \exp \left[2 d_{\mathfrak{L}}(f(s), f(s=0))\right]\right|_{0} ^{\ell}\right] \\
& =\frac{1}{\kappa_{\mathfrak{L}}(\mathcal{A})}\left[\frac{1}{2}\left[\exp \left(2 \mathrm{~d}_{\mathfrak{L}}(\mathcal{A}, \ell)\right)-\exp \left(-2 \mathrm{~d}_{\mathfrak{L}}(\mathcal{A}, 0)\right)\right]\right] \\
& =\frac{1}{\kappa_{\mathfrak{L}}(\mathcal{A})}\left[\frac{1}{2}\left[\exp \left(2 \mathrm{~d}_{\mathfrak{L}}(\mathcal{A}, \ell)\right)-1\right]\right] \\
|f(z)| & \leq \frac{1}{2 \kappa_{\mathfrak{L}}(A)}\left[\exp \left(2 \mathrm{~d}_{\mathfrak{L}}(\mathcal{A}, \ell)\right)-1\right]
\end{aligned}
$$

Here, $\kappa_{\mathfrak{L}}(A)=\lambda_{\mathfrak{L}}(0)=\frac{1}{\left|w^{\prime}(s)\right|}=\frac{1}{\left|f^{\prime}(0)\right|}=1$, with the fact that

$$
e^{2 d_{\mathfrak{R}}(A, L)}=e^{2\left[\frac{1}{2} \log \frac{1+|z|}{1-|z|}\right]}=\frac{1+|z|}{1-|z|}
$$

so that $e^{2 d_{\mathfrak{R}}(A, L)}-1=\frac{1+|z|}{1-|z|}-1=\frac{1+|z|-1+|z|}{1-|z|}=\frac{2 z}{1-|z|}$, which implies

$|f(z)| \leq \frac{1}{2(1)} \cdot \frac{2|z|}{1-|z|}=\frac{|z|}{1-|z|}=\frac{\mathfrak{r}}{1-\mathfrak{r}}$, as required

Theorem (2.3). If $f$ is a holomorphic and convex function defined on a convexity region $\mathfrak{L}$, then $\left|\frac{z f^{\prime}(z)}{f(z)}\right| \geq \frac{1}{1+r}$, where $|z|=r, z \in \mathbb{C}$.

Proof. Given $f$ is a univalent function, that is $f \in \mathcal{S}$, let $f \in \mathcal{S}$ and $\xi \in \mathfrak{D}$ such that, $\mathcal{F}(z)=$ $\frac{f\left(\frac{z+\xi}{1+\xi}\right)-f(\xi)}{\left(1-|z|^{2}\right) f^{\prime}(\xi)}$

Suppose that $z=-\xi$ belongs to $\mathfrak{D}$, then $\mathcal{F}(z)$ reforms to

$$
\begin{aligned}
\mathcal{F}(-\xi)=\frac{f\left(\frac{-\xi+\xi)}{1+\bar{\xi} \xi}\right)-f(\xi)}{\left(1-|\xi|^{2}\right) f^{\prime}(\xi)} & =\frac{f(0)-f(\xi)}{\left(1-|\xi|^{2}\right) f^{\prime}(\xi)} \\
& =\frac{-f(\xi)}{\left(1-|\xi|^{2}\right) f^{\prime}(\xi)} .
\end{aligned}
$$

We apply the preceding theorem (2.2) to a function that has a convexity property in addition to a univalent property, in order to have

$$
\begin{gathered}
|\mathcal{F}(-\xi)| \leq \frac{|\xi|}{1-|\xi|} \\
\left|\frac{f(\xi)}{\left(1-|\xi|^{2} f^{\prime}(\xi)\right.}\right| \leq \frac{|\xi|}{1-|\xi|} \\
\frac{\left(1-|\xi|^{2}\right)}{|\xi|}\left|\frac{f(\xi)}{\left(1-|\xi|^{2} f^{\prime}(\xi)\right.}\right| \leq \frac{|\xi|}{1-|\xi|} \frac{\left(1-|\xi|^{2}\right)}{|\xi|}
\end{gathered}
$$




$$
\begin{aligned}
& \left|\frac{f(\xi)}{\xi f^{\prime}(\xi)}\right| \leq(1+|\xi|) \\
& \left|\frac{\xi f^{\prime}(\xi)}{f(\xi)}\right| \geq \frac{1}{1+|\xi|}
\end{aligned}
$$

We replace $z$ instead of $\xi$ to obtain $\left|\frac{z f^{\prime}(z)}{f(z)}\right| \geq \frac{1}{1+|z|}$

Theorem (2.4) Let $f: \mathfrak{D} \rightarrow \mathfrak{L}$ be a holomorphic and hyperbolic univalent convex function in the unit disk $\mathfrak{D}$. Then, the domain $\mathfrak{L}$ is hyperbolic convex if

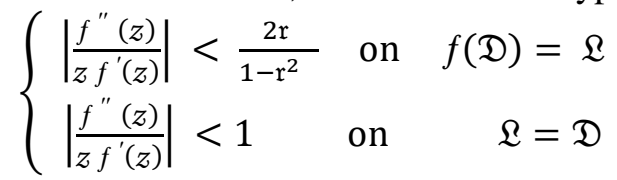

\section{Short Structure of facts}

Let $f: \mathfrak{D} \rightarrow \mathfrak{L}$ be a holomorphic and hyperbolic univalent convex function. Then, for any $z_{1}, z_{2} \in$ $\mathfrak{D}$, the closed geodisc arc $\gamma_{1}$ is joining the points $z_{1}, z_{2}$.

In this proof, we have two important cases,
i. $\quad \mathfrak{L}=f(\mathfrak{D})$.
ii. $\quad \mathfrak{L}=\mathfrak{D} ;($ in case $f: \mathfrak{D} \rightarrow \mathfrak{D})$.

Proof. i- For $\mathfrak{L}=\boldsymbol{f}(\mathfrak{D})$.

Since $f$ a is hyperbolic univalent convex function, and for $z \in \mathfrak{D}$, and $\mathfrak{r}>0$ is radius of an open disk $\mathfrak{D}$ centered at 0 , which is contained in $f(\mathfrak{D})$,

then $\kappa_{\mathfrak{L}}[f(z)]\left|f^{\prime}(z)\right|=\frac{1}{1-\mathrm{r}^{2}}$

Here, we have to show that $f$ must map each subdisk $|z|<\mathfrak{r}$ onto the hyperbolic region $f(\mathfrak{D})$.

The Gaussian curvature should be used in logarithmic cases to do this, as follows:

$$
\begin{aligned}
\log \left[\Lambda_{\mathfrak{L}}[f(z)]\left|f^{\prime}(z)\right|\right]=\log \frac{1}{1-\mathfrak{r}^{2}} . \\
\log \Lambda_{\mathfrak{L}}[f(z)]+\log \left|f^{\prime}(z)\right|=-\log \left(1-\mathfrak{r}^{2}\right)
\end{aligned}
$$

Suppose that $\gamma_{1}: z \rightarrow z(t), t \in I$ where $I$ is an interval on the $\mathrm{x}$-axes, in order to derive (2.5) with respect to the unit normal $\mathcal{N}(z)$ at $z$ that makes a right angle with tangent vector to $\gamma$ at $z$, as follows:

$$
\frac{\partial}{\partial \mathcal{N}} \log \Lambda_{\mathfrak{L}}[f(z)]+\frac{\partial}{\partial \mathcal{N}(z)} \log |f(z)|^{\prime}=-\frac{\partial}{\partial \mathcal{N}(z)} \log \left(1-|z|^{2}\right)
$$

From the hyperbolic metric $\kappa_{\mathfrak{L}}(f(z))\left|f^{\prime}(z)\right|=\frac{1}{1-\mathrm{r}^{2}}$ and the formula below

$h(z, \gamma) \kappa_{\mathfrak{L}}(z)=-\frac{\partial \log \Lambda_{\mathfrak{R}}(z)}{\partial \mathcal{N}(z)} \quad(\mathrm{cf} .[1])$

we obtain,

$$
-2 \mathfrak{J}\left[\frac{\partial}{\partial z} \log \Lambda_{f(\mathfrak{D})}[f(z)] \cdot \frac{z^{\prime}}{\left|z^{\prime}\right|}\right]+\frac{\partial}{\partial z} \log \left|f^{\prime}\right| \cdot \frac{z^{\prime}}{\left|z^{\prime}\right|}=-\frac{\partial}{\partial z} \log \left(1-\mathfrak{r}^{2}\right) \cdot \frac{z^{\prime}}{\left|z^{\prime}\right|} \ldots
$$

It is worth to note that the logarithmic partial derivative has a role to extend the open disk $\mathcal{D}=$ $\left\{w:\left|\frac{w-\mathfrak{r} e^{i \varphi}}{1-e^{-i \varphi_{w}}}\right|<\mathfrak{r}\right\}$, which is hyperbolic convex in $\mathfrak{L}$ for all and larger in $f(\mathfrak{D})$, which makes ( 2.6 ) to be as follow .

$-2 \mathfrak{J}\left[\frac{\partial}{\partial z} \log \Lambda_{f(\mathcal{D})}[f(z)] \cdot \frac{w^{\prime}}{\left|w^{\prime}\right|}\right]+\frac{\partial}{\partial z} \log \left|f^{\prime}(z)\right| \cdot \frac{w^{\prime}}{\left|w^{\prime}\right|}=-\log \left(1-\mathfrak{r}^{2}\right) \cdot \frac{w^{\prime}}{\left|w^{\prime}\right|}$

Let $r \rightarrow 0$ in the larger unit disk $\mathcal{D}$, to have

$$
\begin{aligned}
-2 \mathfrak{I}\left[\frac{\partial}{\partial z} \log \Lambda_{f(\mathfrak{D})}[f(z)] \cdot \frac{w^{\prime}}{\left|w^{\prime}\right|}\right]=\left(-\frac{\partial}{\partial z} \log \left|f(z)^{\prime}\right|\right) \cdot \frac{w^{\prime}}{\left|w^{\prime}\right|} \\
\mathfrak{J}\left[\frac{\partial}{\partial z} \log \Lambda_{f(\mathfrak{D})}[f(z)] \cdot \frac{w^{\prime}}{\left|w^{\prime}\right|}\right]=\left(\frac{\partial}{\partial z} \log \left|\frac{f^{\prime}(z)}{2}\right|\right) \cdot \frac{w^{\prime}}{\left|w^{\prime}\right|}
\end{aligned}
$$

The right - side of (2.7) will be limited to reduce to the state $\left(\frac{\partial}{\partial z} \log \left|\frac{f^{\prime}(z)}{2}\right|\right) \cdot \frac{w^{\prime}}{\left|w^{\prime}\right|}=\frac{1}{2}\left|\frac{f(z)^{\prime \prime}}{f^{\prime}(z)}\right|$ on $\mathfrak{D}$ with $\frac{w^{\prime}}{\left|w^{\prime}\right|} \rightarrow 1$. 
Such that $\left|\frac{f(z)^{\prime \prime}}{2 f^{\prime}(z)}\right|<\frac{2 r}{1-\mathfrak{r}^{2}}$.

ii $-\mathfrak{L}=\mathfrak{D}$

Since $f$ is a hyperbolic univalent convex function defined on the open disk $\mathfrak{D}$ which is centered at 0 and $\mathfrak{r}>0$, then $\mathfrak{D}$ can be contained in $f(\mathfrak{D})$, with $\kappa_{\mathfrak{L}}[f(z)]\left|f^{\prime}(z)\right|=\frac{1}{1-\mathrm{r}^{2}}$

Here, we have to show that $f$ must map each subdisk $|z|<\mathfrak{r}$ onto the hyperbolic region $f(\mathfrak{D})$. The Gaussian curvature should be used in logarithmic cases to achieve this aim, as follows:

$$
\log \left[\Lambda_{\mathfrak{L}}[f(z)]\left|f^{\prime}(z)\right|\right]=\log \frac{1}{1-\mathfrak{r}^{2}} .
$$

Suppose that $\gamma_{1}: z \rightarrow z(t), t \in I$, where $I$ is an interval on the $\mathrm{x}-$ axes, in order to derive (2.8) with respect to the unit normal $\mathcal{N}(z)$ at $z$ that makes a right angle with the tangent vector to $\gamma$ at $z$, as follows:

$\frac{\partial}{\partial \mathcal{N}} \log \Lambda_{\mathfrak{L}}[f(z)]+\frac{\partial}{\partial \mathcal{N}(z)} \log |f(z)|^{\prime}=-\frac{\partial}{\partial \mathcal{N}(z)} \log \left(1-|z|^{2}\right)$

From the hyperbolic metric $\kappa_{\mathfrak{L}}(f(z))\left|f(z)^{\prime}\right|=\frac{1}{1-\mathrm{r}^{2}}$ and the formula below

$h(z, \gamma) \kappa_{\mathfrak{L}}(z)=-\frac{\partial \log \kappa_{\mathfrak{L}}(z)}{\partial \mathcal{N}(z)}$,

we obtain

$$
\begin{gathered}
-2 \Im\left[\frac{\partial}{\partial z} \log \Lambda_{f(\mathfrak{D})}[f(z)] \cdot \frac{z^{\prime}}{\left|z^{\prime}\right|}\right]+\frac{\partial}{\partial z} \log \left|f^{\prime}\right| \cdot \frac{z^{\prime}}{\left|z^{\prime}\right|}=-\frac{\partial}{\partial z} \log \left(1-\mathfrak{r}^{2}\right) \cdot \frac{z^{\prime}}{\left|z^{\prime}\right|} \\
-2 \Im\left[\frac{\partial}{\partial z} \log \Lambda_{\mathfrak{L}}[f(z)] \cdot \frac{z^{\prime}}{\left|z^{\prime}\right|}\right]+\frac{\partial}{\partial z} \log \left|f^{\prime}\right| \cdot \frac{z^{\prime}}{\left|z^{\prime}\right|}=-\frac{\partial}{\partial z} \log \left(1-\mathfrak{r}^{2}\right) \cdot \frac{z^{\prime}}{\left|z^{\prime}\right|}
\end{gathered}
$$

Hence, when $\mathfrak{L}=\mathfrak{D}$, we have

$-2 \mathfrak{I}\left[\frac{\bar{z} z^{\prime}}{\left|z^{\prime}\right|}\right]+\left|\frac{f(z)^{\prime \prime}}{f^{\prime}(z)}\right| \cdot \frac{z^{\prime}}{\left|z^{\prime}\right|}=-\frac{\partial}{\partial z} \log \left(1-\mathfrak{r}^{2}\right) \cdot \frac{z^{\prime}}{\left|z^{\prime}\right|}$

Let $r \rightarrow 0$ such that $-2 \mathfrak{J}\left[\frac{\bar{z} z^{\prime}}{\left|z^{\prime}\right|}\right]+\left|\frac{f(z)^{\prime \prime}}{f(z)^{\prime}}\right| \cdot \frac{z^{\prime}}{\left|z^{\prime}\right|}=0$.

$\mathfrak{I}\left[\frac{\bar{z} z^{\prime}}{\left|z^{\prime}\right|}\right]=\left|\frac{f(z)^{\prime \prime}}{2 f^{\prime}(z)}\right| \cdot \frac{z^{\prime}}{\left|z^{\prime}\right|}$ on $=\mathfrak{D}$, with $\frac{z^{\prime}}{\left|z^{\prime}\right|} \rightarrow 1$, in order to get $\left|\frac{f(z)^{\prime \prime}}{2 f(z)^{\prime}}\right| \cdot \frac{z^{\prime}}{\left|z^{\prime}\right|}=\frac{1}{2}\left|\frac{f(z)^{\prime \prime}}{f^{\prime}(z)}\right|$

$\mathfrak{L}=\mathfrak{D}$. So, the required $\left|\frac{f(z)^{\prime \prime}}{2 f^{\prime}(z)}\right|<1$ is satisfied.

From (i) and (ii), the proof is complete

\section{Conclusions}

The deformation properties of convex and univalent functions in the determination of the relationship between the first and second derivatives of the given function, on one hand, and their association with the range of the region $\mathfrak{I}$ with convex properties, on the other, can be adopted.

As a consequence of the above conclusion, the distortion property was adopted in the proof of the upper bound of the convex and univalent function.

Another estimate in theorem (2.4) was obtained by combining both the distortion and growth characteristics to clarify the effect of the state disk image on the formulation of estimating inequalities that guarantee that the function preserves its geometric and analytical properties.

\section{Acknowledgments}

The authors would like to express deep thanks and gratitude to the Department of Mathematics , College of Science, Mustansiriyah University for their remarkable support to this research.

\section{References}

1. Seong - A Kim.1995. "On Convex Hyperbolic Regions". Japan . J . Math, 23(1): 157 - 162.

2. Shatha S. Alhily, "Higher integrability of the gradient of conformal maps", PhD Thesis, Sussex University, 2013.

3. Peter L. Duren. 1983. "Univalent Functions". Springer - Verlag, New York, Berlin Heidelberg Tokyo, Dept. Math University of Michigan. 
4. Haneen Abbas Saleh and Shatha S Alhily.2020. "Growth and Bounded Solution of Second-Order of Complex Differential Equations Through of Coefficient Function", IOP Conference Series: Materials Science and Engineering, 871(1).

5. Rafie Habib Buti and Kassim A. Jassim. 2019. "On a Class of W- Valent Functions with Two Fixed Points Involving Hypergeomatric Function with Generalization Integral Operator", Iraqi Journal of Science, 60(8): 1753-1759.

6. Sattar Kamil Hussein and Kassim Abdulhameed Jassim . 2019. On a Class of Meromorphic Multi valent Functions Convoluted with Higher Derivatives of Fractional Calculus Operator", Iraqi Journal of Science, 60(10): 2237-2245.

7. Minda, C.D. 1987. A reflection Principle for the Hyperbolic Metric and Applications to Geometric Function Theory", Complex Variables Theory Appl. 8: 129-144.

8. Wan Gang Ma and David Mind. 1994. "Hyperbolically convex functions", Ann Polon. Math., 60(1): 81-100.

9. S. Yamashita.1990. "Convexity Inequalities Differentiable Concernant Density Hyperbolic", C. R. Acad. Sci. Paris, ser. I, 311: $781-78$.

10. T. Sheil -Small. 1969. "On convex univalent functions". J. London . Math . Soc. 2(1): 483-492.

11. Roger W. Barnard, Leah B. Cole, Kent Pearce, and G. Brock Williams,. 2003. Sharp Bounds for the Schwarzian Derivative for Hyperbolically Convex Functions".

12. S.Kim and D. Minda. 1994. "Two-point Distortion Theorem for Univalent Functions". Pacific J. Math., 16(3): 137 - 157.

13. Diego Mejia and Ch. Pommerenke.2000. "On hyperbolically convex functions". J. Geom . Anal., 10(2): 365-378, 2000.

14. Shatha S. Alhily. 2017. "Weighted composition operator on convexity of Bergman spaces", Mathematics and Statistics Journal, 3(2).

15. Alan. F. Beardon and D. Minda. 2007. "The Hyperbolic Metric and Geometric Function Theory",Proc. of the Inter. Workshop on (IWQCMA05), Narosa Publisher, 956.

16. Thomas, G., Weir, M., Hass, J. ,Thomas. 2014.' Calculus: Early Transcendental", 13th ed., New York: Pearson; 2014. 